\title{
Subsidiary adaptations: climbing techniques in vespertilionid bats
}

\section{Olga Emelianova ${ }^{1}$, Aleksandra Panyutina², Ksenia Morozova1, Yakov Davidov' ${ }^{1}$ Maria Kovalenko', Daria Kalacheva', and Anastasia Shvyrkova ${ }^{1}$}

${ }^{1}$ Faculty of Biology, M. V. Lomonosov Moscow State University, Vorobjevy Gory, 1/12, Moscow, 119991, Russian Federation

${ }^{2}$ A. N. Severtsov Institute of Ecology and Evolution, Russian Academy of Sciences, Leninskij Prospekt, 33, 119071, Moscow, Russian Federation

Address correspondence and requests for materials to Aleksandra Panyutina, myotis@mail.ru

Citation: Emelianova, O., Panyutina, A. Morozova, K., Davidov, Y, Kovalenko, M. Kalacheva, D., and Shvyrkova, A. 2019. Subsidiary adaptations: climbing techniques in vespertilionid bats. Bio. Comm. 64(1): 20-30. https://doi.org/10.21638/ spbu03.2019.103

Author's information: Olga

Emelianova, Graduate student, orcid. org/0000-0002-7656-2961; Aleksandra Panyutina, PhD, Senior Researcher, orcid.org/0000-0002-8379-8526; Ksenia Morozova, Undergraduate student, orcid.org/0000-0002-9074-6038; Yakov Davidov, Undergraduate student, orcid. org/0000-0002-0851-1548: Maria Kovalenko, Undergraduate student, orcid. org/0000-0002-9431-112X; Daria Kalacheva, Undergraduate student, orcid.org/00000002-4600-8134; Anastasia Shvyrkova, Undergraduate student, orcid.org/00000002-8742-6295

Manuscript Editor: Pavel Skutschas, Department of Vertebrate Zoology, Faculty of Biology, Saint Petersburg State University, Saint Petersburg, Russia

Received: February 21, 2019;

Revised: April 3, 2019;

Accepted: April 6, 2019;

Copyright: (c) 2019 Emelianova et al. This is an open-access article distributed under the terms of the License Agreement with Saint Petersburg State University, which permits to the authors unrestricted distribution, and self-archiving free of charge.

Funding: The data processing was fulfilled with the support of RFBR (17-04-00954 A).

Competing interests: The authors have declared that no competing interests exist.

\section{Abstract}

The quadrupedal locomotion of bats still remains almost unexplored. Meanwhile, in the life of many species, this type of movement plays an important role. This paper presents the study of characteristics of quadrupedal locomotion on vertical surfaces in bats. We provide the results of the analysis of climbing of five vespertilionid species. The study is based on high-speed video recording of locomotion in two planes in an experimental enclosure on various substrates (the bark of five tree species and the inner surface of the manufactured nest box for bats). For comparison, we used data on horizontal locomotion obtained using the same experimental facilities, as well as all available literature data to date. The time, metric and velocity characteristics of the movement of different species representatives are determined. We show how these characteristics are interrelated and how they differ in walking or climbing. The study comparing walking vs. climbing was performed for the second time ever. It is shown that while climbing, bats retain the same limb sequence, but the gait is not a slow trot-like walk but a very slow walk. There are considerably fewer deviations from the usual symmetrical sequence gait (wherein a forelimb movement is followed by that of the contralateral hind limb) when climbing than when walking, and they are all associated with the search for support. Our results show that not all tree-dwelling species of vesper bats can move up the trees which they use to roost. The surface of the inner walls of artificial shelters for bats may be a significant factor in their occupancy. While designing such shelters, it is advisable to consider the results of our experiments or conduct tests on the behavior of particular species on specific artificial surfaces.

Keywords: climbing, terrestrial locomotion of bats, quadrupedal locomotion, gait analysis, metric walking characteristics.

\section{Introduction}

Bats are the only mammals that mainly use flapping flight to move around. Other types of locomotion are either secondary to them or entirely absent. In some groups of bats, the ability to walk or climb on solid surfaces was lost as a result of the acquisition of mechanical rigidity in the ribcage, up to complete fusion of the ribs in the case of extreme specialization (Kovtun, 1984; Panyutina et al., 2018), which allows the bats to save muscle energy during flight. These groups include the horseshoe bats, the Old World leaf-nosed bats, a significant part of the New World leaf-nosed bats and, to a lesser extent, the funnel-eared bats (Vaughan, 1970). However, the rigidity of the ribcage leads to the inability of such bats to climb, since the capacity to bend the spine is lost. As a result, the variety of places they can use as shelters is strictly limited. As a rule, chiropterans with rigid ribcages mainly inhabit karst 
caves with wide entrances, where they can fly in and land directly on the spot of their choosing (Kuzyakin, 1950). Their locomotion at the roosting places is confined to small movements on the cave ceiling on their hind limbs while hanging upside down.

Unlike chiropterans with rigid ribcages, vesper bats are perfectly able to walk and climb on solid surfaces using all the four limbs. This type of locomotion is their key adaptation as it allows them to inhabit shelters with small entrances like those in tree trunks, man-made buildings, nest boxes, cracks in quarry walls, and others.

It is commonly known that vesper bats mainly use the symmetrical gait moving their limbs in the following order: left forelimb - right hind limb - right forelimb - left hind limb, and so on. This order was termed "the diagonal sequence" by Gray (1944) but "the lateral sequence" by Hildebrand (1965), and we will keep Gray's terminology. However, there is some evidence of deviations from this sequence in bats (Lawrence, 1963; Adams and Carter, 2017; Emelianova et al., 2018). At the moment, there are only two studies dedicated to climbing locomotion of chiropterans. The first one (Riskin, 2000) concerns the Spix's disk-winged bat (Thyroptera tricolor), which was shown to climb smooth plant leaves with the help of its attachment disks employing the slow pace (the left hind limb was extended anteriorly at the same time as the left forelimb, and the right hind limb with the right forelimb). The second study, which is more relevant for us, is based on the comparison of the quadrupedal locomotion in Microchiroptera and Megachiroptera (Adams and Carter, 2017). Experiments were carried out with five species of fruit bats (Eidolon helvum, Rousettus aegyptiacus, Pteropus hypomelanus, Pteropus pumilus, Cynopterus brachyotis), as well as with microbats presented by three species of Vespertillionidae ( $M y$ otis thysanodes, Eptesicus fuscus, Nyticeius humeralis) and one species of Phyllostomidae (Artibeus jamaicensis). The authors investigated both horizontal walking and vertical climbing of these species. They measured the duration of the locomotory cycle (stride) and some skeletal angles. All investigated bats climbed with the diagonal-sequence symmetrical gait (LF-RH-RF-LH) (Adams and Carter, 2017: Fig. 7). The authors noted that megachiropterans initiated movement by a hind limb, while microchiropterans initiated the movement by a forelimb. Also we noticed the pace in the climbing Eidolon helvum (Adams and Carter, 2017: S1 Video.).

Adams and Carter (2017) emphasized that megachiropterans cycled their limbs significantly faster when climbing than when attempting to walk, whereas microchiropterans cycled their limbs at significantly faster rates when walking than when climbing. It should be noted that the experimental study of climbing was performed on a rigid plastic net with a mesh size of $12.7 \times 12.7 \mathrm{~mm}$. Since animals on such a net have a wide range of available footholds, it can be assumed that their movements are not constrained by the substrate making it easy to climb.

However, in the course of their research, our colleagues (Adams and Karter, 2017) did not study the speed of climbing and walking. Therefore, we can make a comparison only with respect to the duration of locomotory cycles, as well as calculate a small number of parameters based on the figures provided in the supplement. Thus, in the literature there is practically no suitable material for comparing vertical and horizontal locomotion of bats.

The goal of this study was to investigate the climbing of vesper bats on different types of wooden substrates that they come across in their daily lives, as well as to determine whether features that are characteristic for horizontal locomotion (Emelianova et al., 2018) are also retained in climbing. As our research was largely reconnaissance, we outlined a wide range of questions that we wanted to get at least preliminary answers:

- What are the features (speed, limb sequence and gait, width and length of steps) of vertical climbing in vesper bats?

- What are the trees in Central Russia that the vesper bat can climb?

- Are different tree species equally suitable for bats?

- Do different species of bats climb trees equally well?

- Are there any links between the ability to climb different trees with the overall ecological characteristics of a bat species?

To reach the goal outlined above, the following key tasks of experiments were identified: to figure out the gait vesper bats use for climbing; to measure the speed of climbing and the characteristics of steps of five bat species on six tree samples and compare the results to the data obtained for horizontal locomotion; and to check for possible interconnections between the measured characteristics and known ecological features of the species in question.

\section{Materials and Methods}

Our research focused on five species of vesper bats found in the Moscow Region, namely the common noctule (Nyctalus noctula), the parti-coloured bat (Vespertilio murinus), the pond bat (Myotis dasycneme), the Daubenton's bat (M.daubentonii), and the Nathusius' pipistrelle (Pipistrellus nathusii). The choice of these species was determined by the fact that all these species tend to occupy shelters with small entrances in tree trunks and/or nest boxes (Kuzyakin, 1950). Thus, we can assume that locomotion on wood surfaces plays a significant role in their life. However, little is known of the characteristics of this locomotion. 


\section{BAT CAPTURE}

The bats were captured in July near the water bodies of the Odintsovo District of the Moscow Region. Mobile traps and mist nets were used for trapping. Most of the animals were born in the current year; nevertheless, all of them were able to climb and fly properly, since they were caught away from their shelters (Table 1). During the experiment the captured bats were kept in artificial shelters on the territory of the biomechanical facility of Skadovsky Zvenigorod Biological Station of Moscow State University (ZBS MSU, Moscow Region). All the animals were released in their natural habitat after the experiment. Capture, maintenance and the protocol of the experiment were approved by the bioethical commission of the A. N. Severtsov Institute of Ecology and Evolution (SIEE RAS), meeting protocol № 1-2017. During maintenance the animals were nourished properly, and weighing showed that during captivity, the body mass of the bats changed insignificantly; such fluctuations were due to natural causes.

Table 1. List of the species analyzed and the quantity of specimen

\begin{tabular}{l|c|c}
\hline \multicolumn{1}{c|}{ Species } & $\begin{array}{c}\text { Number of } \\
\text { specimen and age }\end{array}$ & $\begin{array}{c}\text { Forearm } \\
\text { length (R), mm }\end{array}$ \\
\hline Nyctalus noctula & $3 \mathrm{sad}$ & $53,5 / 54 / 55$ \\
\hline Vespertilio murinus & $1 \mathrm{sad}, 1 \mathrm{ad}$ & $44,8 / 45,5$ \\
\hline Myotis dasycneme & $2 \mathrm{sad}$ & $44 / 46,1$ \\
\hline Myotis daubentonii & $2 \mathrm{ad}$ & $34,4 / 37,4$ \\
\hline Pipistrellus nathusii & $3 \mathrm{sad}$ & $32,9 / 33 / 34,5$ \\
\hline
\end{tabular}

\section{EXPERIMENTAL DESIGN}

To examine the speed of bats' climbing, a vertical corridor was designed and assembled. It consisted of a corridor frame $(20 \mathrm{~cm} \times 15 \mathrm{~cm} \times 70 \mathrm{~cm}$, the last being the height) and interchangeable panels of experimental substrates. The front wall was absent and the panel with a sample of substrate was fitted tightly to the back wall $(20 \mathrm{~cm} \times 70 \mathrm{~cm})$ inside the corridor. One of the side walls was made of transparent plexiglass which allowed filming from the side simultaneously with filming from the front. The second, non-transparent side wall had a scale grid of $10 \mathrm{~cm} \times 10 \mathrm{~cm}$ squares on the inner side (facing the video recorder and making the background for the animal in the side view) for estimating precisely the scale and displacement of the animal in the frame. On the top of the corridor, a shelter was placed that attracted the animal and stimulated it to climb up the substrate. The animal was placed on the substrate at the bottom.

The filming was conducted with two Casio EX F1 cameras at a speed rate of 300 frames per second; the cameras were synchronized with the help of special signals in the frame. For each animal, several passages were recorded on each type of substrate.

\section{EXPERIMENTAL SUBSTRATES}

Six types of substrate were used in the experimental corridor, which included sawed out pieces of wood with bark on it and a standard wall fragment of a nest box made for bats at the Zvenigorod Biological Station in the 1990s (Panyutina, 2014).

Five tree species were sampled for bark: pine tree, spruce, birch, aspen and willow (Table 2, Fig. 1). Aspen bark is the smoothest of all substrates used. Pine bark is distinguished by almost smooth areas stretched in a vertical direction separated by shallow furrows. Spruce bark consists of small $(2-3 \mathrm{~cm})$ rounded scales that often cover the entire surface of the trunk. Willow bark is covered with quite deep, rough edged, mostly longitudinal furrows. There are a lot of gaps in the upper layer of the bark on the surface of the birch trunk; in addition, it is often striated with transverse grooves. The inner surface of the nest box wall represented a smooth plank with sets of 5 shallow horizontal notches repeated every $3 \mathrm{~cm}$. The distance between notches in a set was approximately $1 \mathrm{~cm}$.

\section{VIDEO ANALYSIS}

The resulting video was subjected to primary sorting: video fragments in which the bat stopped on its way to the shelter, climbed very slowly or failed to reach the final destination were rejected.

The initial preparation of videos suitable for analysis included their synchronization and selection of informative fragments in QuickTime and Virtual Dub programs. Detailed frame-by-frame analysis was performed in Virtual Dub and measurements were performed using the Meazure program.

For each bat species, from two to six passages on each substrate were analyzed. Only passages composed of three or more locomotory cycles were included in the analysis.

The movement of animals was analyzed for the limb sequence (based on the results, the diagrams of limb sequences were drawn up for all of the experiments included in the analysis). All deviations from the standard RF-LHLF-RH sequence were noted and analyzed separately in order to determine possible reasons for such irregularities.

In all the records included in the analysis the following parameters were measured:

1. Animal speed. The average speed at which an animal passed through the focal zone of the experimental setup was taken as the animal speed. Its calculation was carried out as follows: vertical distance traveled by a bat on the video was measured in pixels and then it was converted to millimeters by means of calibration (distance between scale squares in millimeters divided by the 


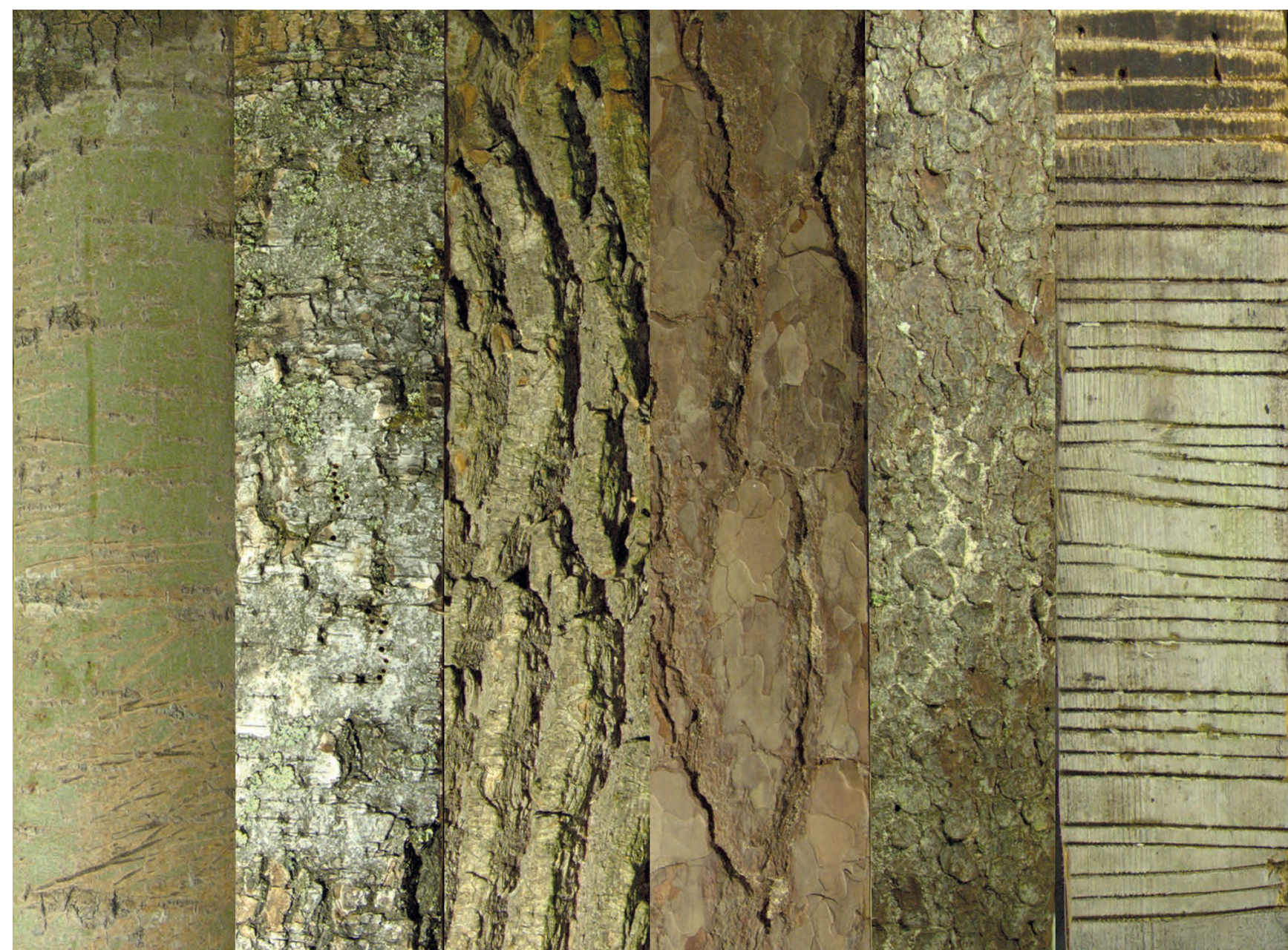

Fig. 1. Substrates used in the experiments. From left to right: aspen bark, birch bark, willow bark, pine bark, spruce bark, and the inner surface of the nest box.

Table 2. Quantity of the material processed (number of specimen / number of transect passages / total number of locomotory cycles)

\begin{tabular}{l|c|c|c|c|c}
\hline Substrate Species & Nyctalus noctula & Vespertilio murinus & Myotis dasycneme & Myotis daubentonii & Pipistrellus nathusii \\
\hline Birch & $1 / 4 / 20$ & $1 / 3 / 16$ & $2 / 3 / 13$ & $1 / 7 / 47$ & $2 / 3 / 22$ \\
\hline Willow & $1 / 3 / 13$ & $2 / 5 / 26$ & $1 / 5 / 22$ & $1 / 4 / 21$ & $1 / 4 / 30$ \\
\hline Pine & $3 / 6 / 30$ & $1 / 5 / 25$ & $2 / 5 / 24$ & $1 / 6 / 35$ & $1 / 5 / 34$ \\
\hline Spruce & $1 / 3 / 13$ & $1 / 4 / 19$ & $1 / 3 / 15$ & $2 / 5 / 30$ & $3 / 6 / 40$ \\
\hline Inner surface of the nest box & $1 / 3 / 15$ & $1 / 2 / 11$ & $1 / 3 / 11$ & $1 / 4 / 21$ & $2 / 5 / 29$ \\
\hline
\end{tabular}

same distance in pixels and multiplied by the measured value). Time in which a bat passed the vertical distance was calculated based on the number of frames between the beginning and the end of the movement. The total formula for speed calculation is:

$$
v=\frac{S_{1} * S_{\text {real }} * k}{S_{\text {pixel }} *\left(C_{2}-C_{1}\right)}
$$

where: $S_{1}-$ distance passed by a bat [pix], $S_{\text {real }}-$ calibration distance $[\mathrm{mm}], S_{\text {pixel }}$ - calibration distance [pix], $C_{1}-$ first frame, $C_{2}-$ final frame, $k-$ filming speed [frame/sec].

Having divided the calculated speed by the forearm length (commonly used dimensional index for chiropterans) we obtained the relative speed (the number of forearm lengths passed per second) - a value which allowed comparison of locomotor abilities of bats irrespective of size differences.

2. Metric characteristics of steps (the length of the locomotory cycle, the fore and hind limbs stance width) were measured and calculated using the same calibra- 
tion method for each locomotory cycle (sequential steps of all four limbs). To calculate the relative values, the measured values were divided by the forearm length.

Since the studied sample is rather small, the obtained data cannot pretend to signify the species locomotion. However, they allow us to give an approximate estimate and delineate the possible range of variations in locomotor characteristics. Therefore, we will rely here on the range of values instead of the averages. The statistical data processing was performed with the Statistica-10 software. The impact of the substrate on the measured parameters was investigated with Kruskal-Wallis ANOVA and Median test $(\mathrm{p}<0,05)$. The coefficients of correlations between parameters were estimated with the Spearman $\mathrm{r}$ criterion for the nonparametric test $(\mathrm{p}<0,05)$.

\section{Results and Discussion}

As a result of our study, we were able to establish the following patterns: first of all, some of the investigated substrates turned out to be unsuitable for the bats' vertical progression. We did not manage to collect any representative data from the experiments with the aspen bark. None of the bats could climb up the aspen: all the tests ended up with the animal either stopping near the start point or climbing up in the corridor corner using the gap between the substrate panel and the side wall of the experimental set as a support. Thus, we exclude all the results of the experiments with the aspen bark from further analysis and consider the vesper bats to be unable to climb it.

\section{LIMB SEQUENCE AND ITS DEVIATIONS}

As we know, bats use the diagonal-sequence symmetrical gait (LF-RH-RF-LH) for climbing, but deviations from it are also observed (Table 3 ). The main reasons for these deviations are:

- searching for support - repeated failures to cling to the substrate with one of the limbs;

- a piece of the substrate breaking off under the clinging limb.

These deviations have specific reasons, contrary to the chaotic deviations which we revealed in horizontal locomotion of bats (Emelianova et al., 2018).

The most convenient substrates for the bats to cling to were the barks of birch and willow, and the wall of the nest box was the least suitable for them. Bats were much more likely to fall from the spruce bark and from the nest box wall, than from the other substrates.

Vespertilio murinus and Myotis dasycneme showed the least number of deviations, while Nyctalus noctula and Pipistrellus nathusii had the most difficulties with climbing, and hence, more deviations from the typical limb sequence.

Importantly, the climbing bats almost never lifted more than one limb at a time, or in other words, the animals tend to clinge to the substrate with at least three limbs, unlike in horizontal locomotion (Emelianova et al., 2018). Among all the passages analyzed, the simultaneous lifting of two limbs was registered only twice (both cases in Myotis dasycneme). So, the gait that vesper bats use for climbing should be specified as a "very slow walk": the three-point and four-point stages of

Table 3. Average number of deviations from the diagonal-symmetrical sequence (ratio of the number of deviations to the number of locomotory cycles) for studied species of bats and for studied types of substrate

\begin{tabular}{|c|c|c|c|}
\hline \multirow{2}{*}{ Substrate } & \multicolumn{2}{|c|}{ Searching for support } & \multirow{2}{*}{$\begin{array}{c}\text { Breaking off } \\
\text { (by forelimb or hindlimb) }\end{array}$} \\
\hline & forelimb & hindlimb & \\
\hline Birch & $1,6 \%$ & $0,3 \%$ & $0,3 \%$ \\
\hline Willow & $0,7 \%$ & $0,1 \%$ & $0,3 \%$ \\
\hline Pine & $4,5 \%$ & $1,5 \%$ & $0,2 \%$ \\
\hline Spruce & $8,5 \%$ & $0 \%$ & $6,0 \%$ \\
\hline Inner surface of the nest box & $58,6 \%$ & $25,3 \%$ & $8,0 \%$ \\
\hline \multirow{2}{*}{ Species } & \multicolumn{2}{|c|}{ Searching for support } & Breaking off \\
\hline & forelimb & hindlimb & (by forelimb or hindlimb) \\
\hline Nyctalus noctula & $86,8 \%$ & $26,4 \%$ & $6,6 \%$ \\
\hline Vespertilio murinus & $27,4 \%$ & $7,6 \%$ & $3,6 \%$ \\
\hline Myotis dasycneme & $34,1 \%$ & $3,5 \%$ & $1,2 \%$ \\
\hline Myotis daubentonii & $34,4 \%$ & $6,5 \%$ & $4,5 \%$ \\
\hline Pipistrellus nathusii & $52,9 \%$ & $23,9 \%$ & $6,5 \%$ \\
\hline
\end{tabular}



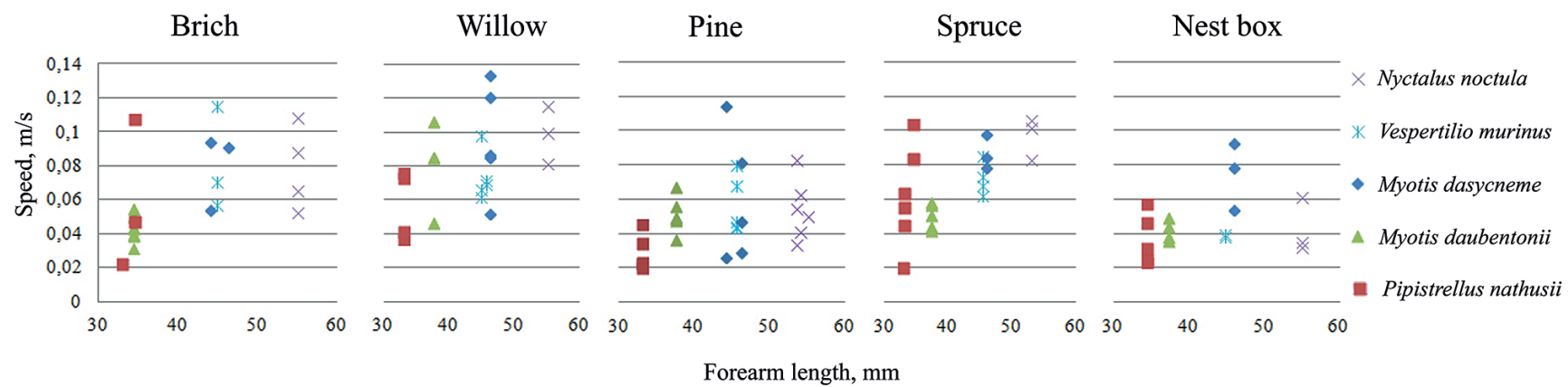

Fig. 2. Variation of the climbing speed on different substrates in the species of bats of different sizes.

support alternate by the formula $4-3-4-3-4-3-4-3$ (Sukhanov, 1968). According to the same terminology, the investigated species of bats use for horizontal locomotion the gait which is a "slow trot-like walk" (2-3-43-2-3-4-3) (Emelianova et al., 2018). On the contrary, the climbing gait of megabats (Adams and Karter, 2017) and the disk-winged bat (Riskin, 2000) can be a "slow pace-like walk" (4-3-2-3-4-3-2-3) or even a slow pace (4-2-4-2).

\section{SPEED MEASUREMENTS}

All the investigated species showed wide ranges of climbing speeds on all the substrates (Fig. 2, Tab. 4). The speed varied from $0.02 \mathrm{~m} / \mathrm{s}$ (Pipistrellus nathusii) to $0.13 \mathrm{~m} / \mathrm{s}$ (Myotis dasycneme). M.dasycneme, M. daubentonii and Nyctalus noctula had the highest speed climbing the willow bark, while Vespertilio murinus and Pipistrellus nathusii had their highest speed climbing the birch.

Myotis daubentonii showed the narrowest range of speeds (except for the experiments with the willow). In other cases, the dispersion varied, but the ranges for different species overlapped. Therefore, we can conclude that there is no clear correlation between the speed and the size of the animal or the speed and the type of sub- strate. The only exception is the wall of the nest box, as all the species climbed it somewhat slower than the natural tree surfaces.

The lack of significant impact of the substrate type on the speed characteristics allowed us to merge the datasets and analyze climbing in general. The maximum recorded values appear to be the most interesting for us in the speed analysis since they characterize the limits of the particular species' capabilities.

The speeds of vertical locomotion turned out to be lower than those of horizontal locomotion, but their ranges more or less overlap in all species (Table 5, Fig. 3). This was particularly pronounced in the case of Myotis daubentonii, which demonstrated the lowest speed values among all the investigated species. For the rest, the bigger bats showed higher speed values (Table 4).

As to the relative speed (velocity divided by the length of the forearm), the distribution of species for this parameter in the horizontal and vertical locomotion had the same order: the fastest species were Myotis dasycneme and Pipistrellus nathusii, the slowest - Nyctalus noctula (Fig. 4). Therefore, a tiny pipistrelle could climb with a very high speed for its size, while a larger noctule moved quite slowly, both when walking and climbing.

Table 4. Speed characteristics

\begin{tabular}{l|c|c|c|c}
\hline \multicolumn{1}{c|}{ Species } & $\begin{array}{c}\text { Maximum speed, } \\
\text { climbing, } \mathbf{m} / \mathbf{s}\end{array}$ & $\begin{array}{c}\text { Minimum speed, } \\
\text { climbing, m/s }\end{array}$ & $\begin{array}{c}\text { Maximum speed, } \\
\text { walking, m/s }\end{array}$ & $\begin{array}{c}\text { Minimum speed, } \\
\text { walking, m/s }\end{array}$ \\
\hline Pipistrellus nathusii & 0,11 & 0,02 & 0,17 & 0,10 \\
\hline Myotis dasycneme & 0,13 & 0,03 & 0,20 & 0,09 \\
\hline Myotis daubentonii & 0,11 & 0,03 & 0,15 & 0,05 \\
\hline Vespertilio murinus & 0,12 & 0,04 & 0,19 & 0,11 \\
\hline Nyctalus noctula & 0,12 & 0,03 & 0,22 & 0,10 \\
\hline Eptesicus fuscus* & - & - & 0,2 & 0,2 \\
\hline Myotis thysanodes* & 0,04 & 0,04 & - & - \\
\hline Mystacina tuberculata** & - & - & 0.95 & 0.13 \\
\hline Desmodus rotundus** & - & - & $\left.1.14^{* * *}\right)$ & $0.12\left(0.28^{* * *}\right)$ \\
\hline
\end{tabular}

* _ from Adams, Carter, 2017; ** — from Riskin et al., 2006; *** — speed during jumping. 


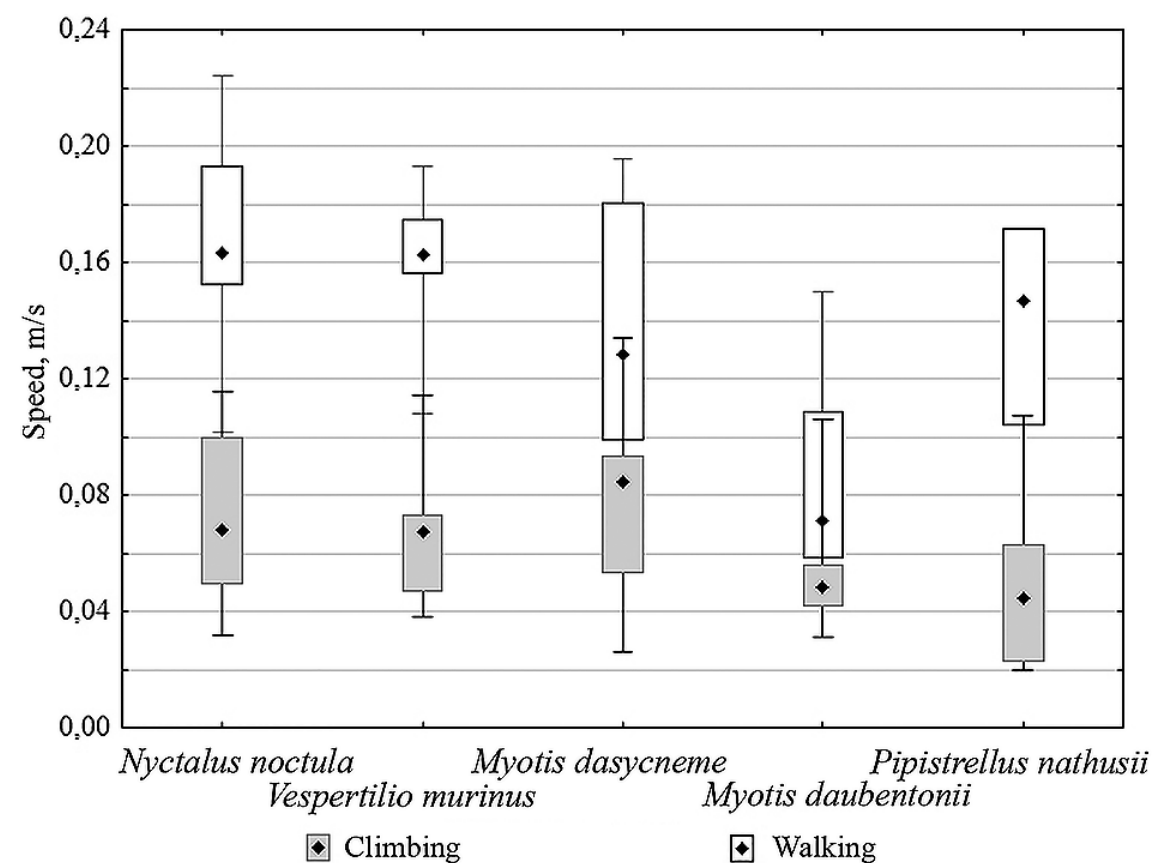

Fig. 3. Walking and climbing speeds in different species of bats. The central square is the median, the rectangle is $25-75 \%$ of the values; the strokes are the maximum and minimum values (hereafter).

Table 5. Maximum and minimum relative speed in studied species during climbing and walking

\begin{tabular}{l|c|c|c|c}
\hline \multicolumn{1}{|c|}{ Species } & $\begin{array}{c}\text { Maximum relative } \\
\text { speed during climbing, } \\
\text { forearm lengths/s }\end{array}$ & $\begin{array}{c}\text { Minimum relative } \\
\text { speed during climbing, } \\
\text { forearm lengths/s }\end{array}$ & $\begin{array}{c}\text { Maximum relative } \\
\text { speed during walking, } \\
\text { forearm lengths/s }\end{array}$ & $\begin{array}{c}\text { Minimum relative } \\
\text { speed during walking, } \\
\text { forearm lengths/s }\end{array}$ \\
\hline Pipistrellus nathusii & 3,12 & 0,60 & 5,2 & 3,17 \\
\hline Myotis dasycneme & 2,90 & 0,60 & 4,45 & 1,89 \\
\hline Myotis daubentonii & 2,84 & 0,91 & 4,35 & 1,52 \\
\hline Vespertilio murinus & 2,56 & 0,86 & 4,25 & 2,41 \\
\hline Nytalus noctula & 2,10 & 0,58 & 4,07 & 1,85 \\
\hline
\end{tabular}

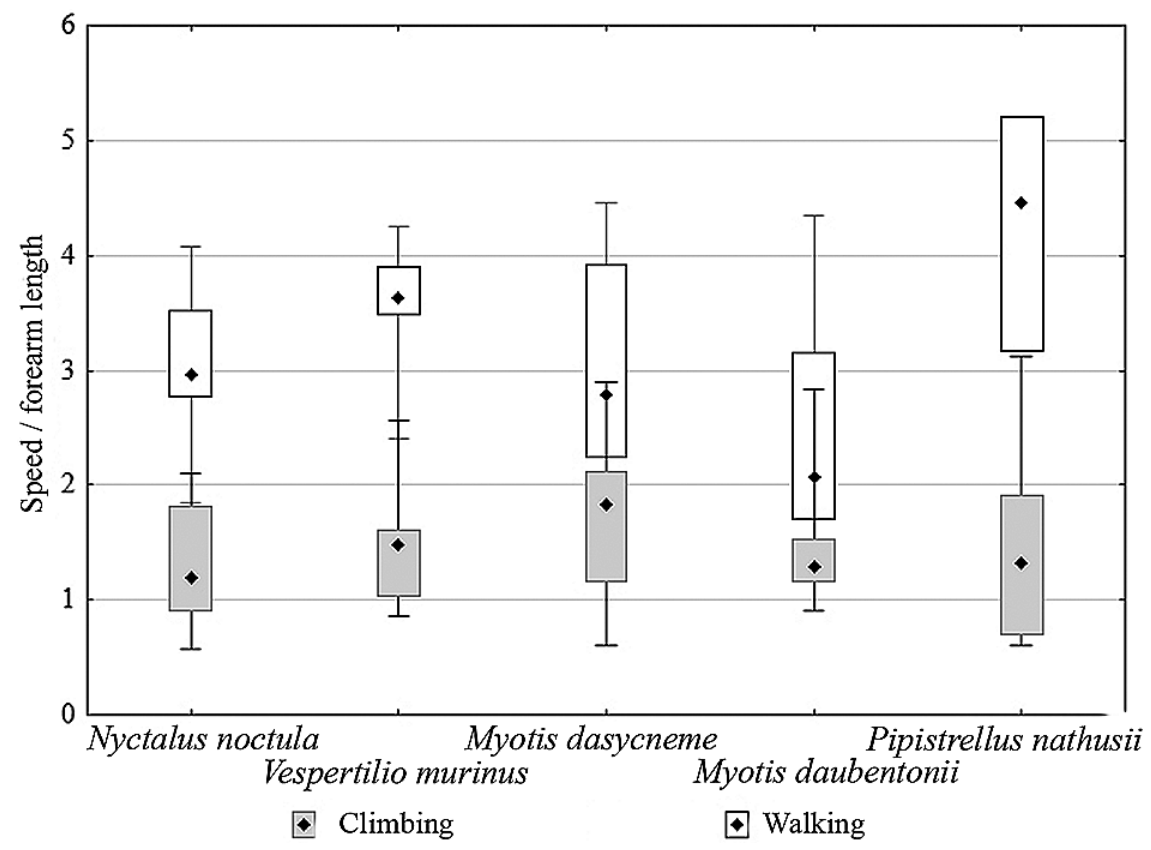

Fig. 4. Relative walking and climbing speeds in different species of bats. 


\section{METRIC MEASUREMENTS}

\section{The length of locomotory cycles}

The highest value of the absolute stride length (i.e., the distance covered over the complete locomotory cycle including the motions of all the four limbs) was naturally found for the larger species - Myotis dasycneme $(99 \mathrm{~mm}$ on the willow) and Vespertilio murinus ( $99 \mathrm{~mm}$ on the nest box wall). Like in speed analysis, we did not find any significant differences in the cycle length on different substrates, so we will further consider the merged dataset.

Basically, the length of the cycle correlates with the size of the animal. However, the significant overlap of the cycle lengths is found among the larger bats (Nyctalus noctula, Myotis dasycneme and Vespertilio murinus), on the one hand, and among the smaller ones (Pipistrellus nathusii and Myotis daubentonii), on the other hand (Fig. 5). The same overlap can be observed in the data for horizontal locomotion.

The relative values (the cycle length divided by the forearm length) are more distinctive. The maximum value of the relative cycle length for Pipistrellus nathusii (2.23 lengths of the forearm) is the highest among all the species, while the maximum value for Nyctalus noctula is the lowest. Except for the leadership of Pipistrellus nathusii, the same order of the species was observed for horizontal locomotion (Fig. 6). Thus, like in the relative speed (see above), Pipistrellus nathusii demonstrated the two highest values, while Nyctalus noctula had the lowest ones.

Comparing this with the data obtained in the study of horizontal locomotion (Emelianova et al., 2018), it can be noted that there are no gaps between the ranges of stride lengths. When climbing, bats make longer strides than while walking, but their overall value range covers the variation of stride lengths while walking, with the exception of the common noctule and the pond bat, whose smallest horizontal stride lengths are far shorter than their vertical stride. Therefore, we can safely assume that bats do not tend to demonstrate peak stride lengths while walking horizontally and keep the stride length in a comfortable range. We can further suggest that longer strides help bats save energy during the ascension, minimizing the limb frequency and maximizing the time period the animal has to search for a next suitable support. While in horizontal progression this problem does not normally arise at all. This explains the increase in the stride length when climbing compared to horizontal locomotion. However, the explanation cannot be attributed to differences in climbing particular substrates, as no significant correlation between the type of surface and the stride length was found, whereas the substrates were specifically selected to provide a wide variety of roughness.

The stable increase in the dispersion of the stride lengths while climbing as compared to walking which was observed in four of the five studied species may have a more serious biomechanical foundation. Moving horizontally, animals that have extremely long limbs in relation to body length, not only the wing-shaped forelimbs but the and hind limbs as well (Panyutina, 2018), can afford to noticeably vary the range of limb movement, choosing the exact range in such a way as to minimize the positive and negative mechanical work of muscles unavoid-

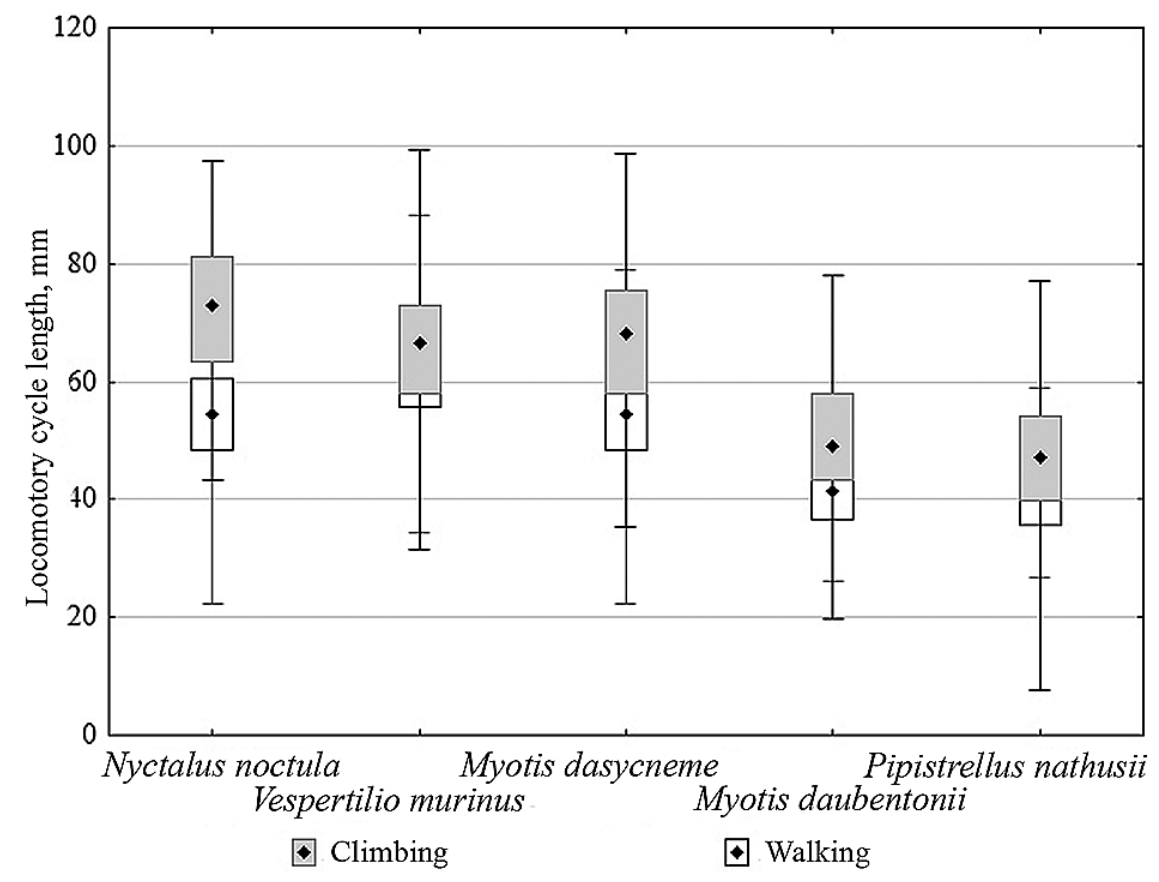

Fig. 5. Lengths of the locomotory cycles during walking and climbing in different species of bats. 


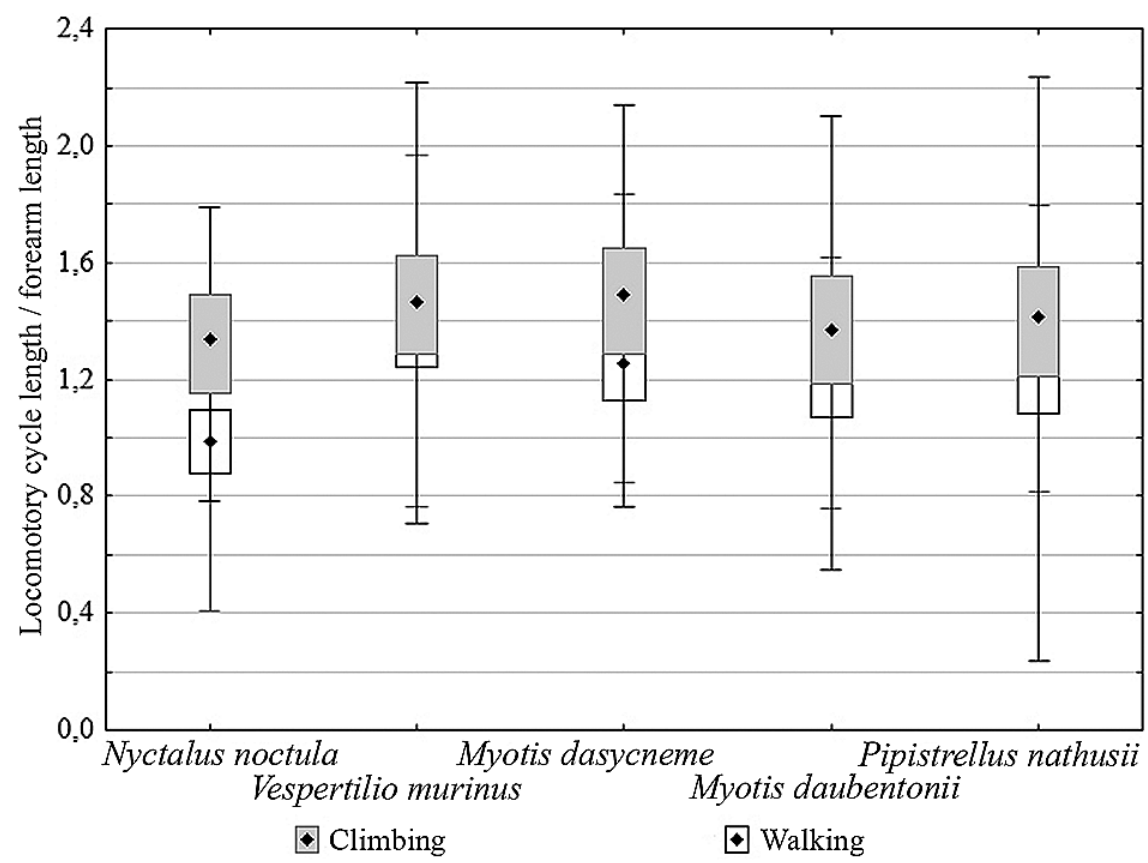

Fig. 6. Relative lengths of the locomotory cycles during walking and climbing in different species of bats.

able in horizontal legged locomotion (Kuznetsov, 2019). Since the limbs of bats are very poorly suited for walking, it is imperative for them in terms of energy saving to use only the most optimal part of the potential span of limb mobility. However, no energy economy is possible when climbing, as the bat performs only the positive mechanical work directly to lift the body and increase its gravitational potential energy, and hence, the energy expended by muscles is strictly proportional to the height increase and the mass of the animal. It means that the range of the stride length is free to be greater than in horizontal walking since there is no energetic benefit in its restriction.

This kind of interpretation needs serious experimental verification in biomechanical experiments with measurement of oxygen consumption. However, as a working hypothesis, it can be preliminary accepted. This opens up a wide field for experimental work on the study of bat endurance in locomotion on horizontal and vertical surfaces.

\section{The limb stance width}

The limb stance width corresponds to the animal's size (the larger the animal, the wider the stance). This trend was retained on all substrates except the pine bark and the nest box wall, where all the species had comparable limb stance width. As with speed measurements, there was no clear difference in the limb stance width between the substrates with the exception of the nest box wall, in which the distance between the forelimbs was generally smaller than that on other substrates. No correlation between the limb stance width of fore and hind limbs was found. The analysis of relative limb stance widths didn't reveal any significant correlations with the type of substrate either.

\section{The duration of locomotory cycles}

The average durations of locomotory cycles were approximately equal for all studied species: $1.1 \mathrm{~s}$ in climbing and $0.4 \mathrm{~s}$ in walking (Table 6, Fig. 7). Interestingly, the cycle duration does not depend on the size of the animal and the substrate, but depends only on the substrate orientation (walking horizontally or climbing vertically).

At first glance, it is quite a surprising conclusion. However, after careful analysis, it becomes clear that this pattern is quite expected. Speed is the result of dividing the distance by its passage time, in this particular case, the length of the cycle by its duration. Therefore, the fact that during vertical climbing the speed is lower (which is not strange, since it requires more mechanical work to be performed per locomotory cycle than on a horizontal substrate) can be explained either by shortening the stride, or by increasing its duration, or both.

In reality, we have seen that the climbing stride, on the contrary, is somewhat longer than the walking stride, so that the difference in the cycle duration between climbing and walking becomes even greater than it would have been if the stride lengths were the same. This feature is more or less kept in all studied species. Therefore, there is an increase in time spent on each stride in climbing as compared to walking. Apparently, this increase is a consequence of the fact that during 


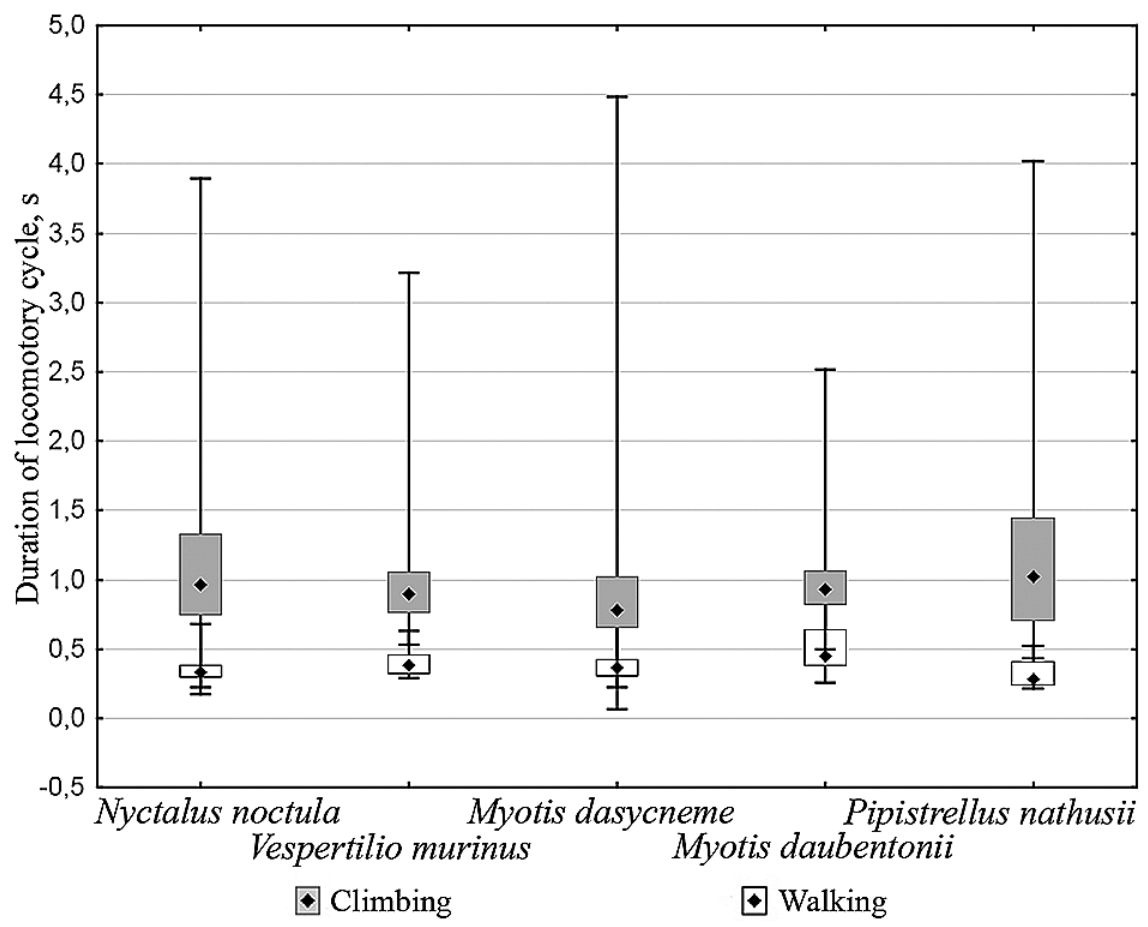

Fig. 7. Durations of the locomotory cycles during walking and climbing in different species of bats.

climbing the energy expenditure is greater compared to horizontal movement over the unit distance. We hypothesize that the 5-fold difference in body weight among the animals studied can hardly have a noticeable effect on the muscular properties, and this is the reason why the average duration of the locomotory cycle is similar in all these species.

The values of average duration of locomotory cycles of the climbing Myotis thysanodes and walking Eptesicus fuscus recalculated from the video data of Adams and Carter (2017) fit the ranges recorded by ourselves (Table 6).

Table 6. Average duration of the locomotory cycle in some species of vespertilionid bats during walking and climbing

\begin{tabular}{l|c|c}
\hline \multicolumn{1}{c|}{ Species } & $\begin{array}{c}\text { Duration of cycle } \\
\text { during walking, s }\end{array}$ & $\begin{array}{c}\text { Duration of cycle } \\
\text { during climbing, s }\end{array}$ \\
\hline Myotis thysanodes* & - & 1 \\
\hline Eptesicus fuscus** & $0,18+/-0,02$ & - \\
\hline Nyctalus noctula & $0,35+/-0,1$ & $1,17+/-0,7$ \\
\hline Vespertilio murinus & $0,40+/-0,1$ & $1,03+/-0,5$ \\
\hline Myotis dasycneme & $0,38+/-0,1$ & $0,98+/-0,8$ \\
\hline Myotis daubentonii & $0,51+/-0,2$ & $0,99+/-0,3$ \\
\hline Pipistrellus nathusii & $0,32+/-0,1$ & $1,21+/-0,7$ \\
\hline
\end{tabular}

* — recalculated from Supplementary video S2 (Adams, Carter, 2017);

** _ recalculated from Supplementary video S5 (Adams, Carter, 2017).

\section{Interrelationship between speed and metric parameters}

The vertical climbing speed in the species that we studied varied 3-5 times (more than when walking horizontally). The increase in speed during climbing, as when walking horizontally (Emelianova et al., 2018), is achieved by increasing the stride frequency, i.e. reduction of the locomotory cycles' duration. In most species/ substrate combinations, we obtained statistically significant negative correlations of speed and cycle duration $(-0.48$ to -0.75$)$, calculated by the Spearman criterion $(\mathrm{p}<0.05)$.

\section{Conclusion}

The results obtained, as well as the previous data (Adams and Carter, 2017) indicate that the speeds of bats moving horizontally exceed those achieved during vertical climbing. According to our measurements the horizontal speeds are about 1.5 times greater, even in the specialized tree-dwellers (Table 5).

Our data do not yet allow us to draw clear conclusions as to which features of the substrate surface are responsible for certain characteristics of chiropteran locomotion. However, on the basis of the conducted experiments, some assumptions regarding their preferences and abilities can be made.

The Nathusius' pipistrelle showed markedly higher relative speed values in climbing and walking, which is 
in good agreement with our knowledge of the ecology of this species. Most often it inhabits attics and other chambers in human buildings or hollows behind loose tree bark. The common noctule, on the other hand, walks and climbs much slower, making overall smaller strides relative to its size. It is this species that mostly prefers living in tree hollows and artificial nest boxes where there is not much space to walk around. The differences between the remaining studied species are too small to be considered reliably.

Notwithstanding the foregoing, it was the common noctule and the Nathusius' pipistrelle that experienced the greatest difficulties during climbing; more often than other species, they spent a long time searching for support and fell from the substrate.

Interestingly enough, aspen bark proved to be unsuitable for the climbing of bats. In central Russia, aspen tree hollows are the most common roosting places for common noctules. Our study shows that when settling in such a shelter the noctule cannot reach it moving along the bark, and the only possible way to get in is to land directly on the edge of the entrance. This might be the reason why noctules so demand the presence of significant open space in front of the entrance to the shelter (Ruczyński and Bogdanowicz, 2005).

The most obvious practical application of our study would be improving the nest box for chiropterans. The inner surface of the nest box wall developed specifically for bats proved to be one of the least favorable substrates to climb. This is particularly clearly seen in the speed characteristics of the three species most likely to inhabit nest boxes (the Nathusius' pipistrelle, the common noctule and the parti-colored bat). Hence, in the manufacture of nest boxes for bats, it is necessary to radically change the approach to surface preparation: instead of sparse and deep transverse grooves, long vertical ones (either straight with notches or meandering) should be made.

\section{Acknowlegments}

We would like to offer our sincere gratitude to S. V Kruskop, A. S. Kruskop and I. V. Artyushin for the help in capturing bats and to A.N. Kuznetsov for constructing the experimental setup. I. B. Soldatova helped us tremendously during filming and analyzing the records. We express our appreciation to V. O. Arzamastseva, P. Y Bikmulina, A. O. Zheludeva, M. A. Zaets, A. A. Kitaytsev, A. I. Molchanova, E.S. Pravdivtseva, N. A. Sivitskaya and N. A. Tikhonova for their assistance during the experiments. E. L. Yakhontov and K. V. Maria contributed greatly to the translation of the article to English.

The experimental part of the study was conducted at the biomechanical facility of S. N. Skadovsky Zvenigorod Biologi- cal Station. The data processing was fulfilled with the support of RFBR (17-04-00954 A).

This study was approved by the Committee of Bio-ethics of the A. N. Severtsov Institute of Ecology and Evolution (research protocol no. 1-2017).

\section{References}

Adams, R. A. and Carter, R. T. 2017. Megachiropteran bats profoundly unique from microchiropterans in climbing and walking locomotion: Evolutionary implications. PLOS ONE 12(9):e0185634. https://doi.org/10.1371/journal. pone.0185634

Emelianova, O.R., Panyutina, A. A., Sivitskaya, N. A., Kitaitsev, A. A., and Tikhonova, N.A. 2018. Spatiotemporal characteristics of quadrupedal locomotion in vespertilionid bats. Biology Bulletin 45(9):999-1011. https://doi. org/10.1134/S1062359018090054

Gray, J. 1944. Studies in the mechanics of the tetrapod skeleton. Journal of Experimental Biology 20(2):88-116.

Hildebrand, M. 1965. Symmetrical gaits of horses. Science 150:701-708. https://doi.org/10.1126/science.150.3697.701

Kovtun, M. F. 1978. Apparat lokomocii rukokrylykh [Locomotor apparatus of bats]. Naukova Dumka, Kiev.

Kuznetsov, A. N. 2018. A theorem of target point for ground reaction force in a planar serial-linked walking limb. Bioinspiration \& Biomimetics 13(6):066010. https://doi. org/10.1088/1748-3190/aae44f

Kuzyakin, A. P. 1950. Letuchie mishi [Bats]. Sovetskaya nauka, Moscow.

Lawrence, M. J. 1969. Some observations on non-volant locomotion in vespertilionid bats. Journal of Zoology 157(3):309317. https://doi.org/10.1111/j.1469-7998.1969.tb01705.x

Panyutina, A. A. 2014. K istorii izucheniya rukokrilih na Zvenigorodskoy biostancii [To the history bat research at the Zvenigorod Biological Station]. Plecotus et al. 17:109-124.

Panyutina, A. A. 2018. Gliding and flapping flight: an alternative or continuity? Zoologichesky zhurnal 97(8):10261040. https://doi.org/10.1134/S0044513418080111

Riskin, D. K. 2000. A behavioural investigation of the sticking mechanisms and non-aerial locomotion of Spix's diskwinged bat, Thyroptera tricolor (Microchiroptera: Thyropteridae). M. Sc. thesis, York University, Toronto.

Riskin, D. K., Parsons, S., Schutt, W. A. Jr., Carter, G. G., and Hermanson, J.W. 2006. Terrestrial locomotion of the New Zealand short-tailed bat Mystacina tuberculata and the common vampire bat Desmodus rotundus. Journal of Experimental Biology 209(9):1725-1736. https://doi. org/10.1242/jeb.02186

Ruczynski, I. and Bogdanowicz, W. 2005. Roost cavity selection by Nyctalus noctula and N. leisleri (Vespertilionidae, Chiroptera) in Bialowieza primeval forest, eastern Poland. Journal of Mammalogy 86(5):921-930. https://doi. org/10.1644/1545-1542(2005)86[921:RCSBNN]2.0.CO;2

Sukhanov, V. B. 1968. Obschaya sistema simmetrichnoy lokomocii nazemnih pozvonochnih I osobennosti peredvizheniya nizshih tetrapod [The general system of the symmetrical locomotion in terrestrial vertebrates and the characteristic features of movement of lower tetrapods]. Nauka, Leningrad.

Vaughan, T.A. 1970. The skeletal system. In: Wimsatt W. A. (ed.) Biology of Bats. Academic Press, New York, 97-138. 